\section{Promoção da saúde, epidemiologia social e capital social: inter-relações e perspectivas para a saúde pública}

\author{
Health promotion, social epidemiology, \\ and social capital: associations and \\ perspectives for public health
}

\author{
1 Secretaria de Estado de \\ Saúde do Distrito Federal, \\ Brasília, Brasil. \\ 2 Centre for Population \\ Studies, London School \\ of Hygiene and Tropical \\ Medicine, England \\ United Kingdon. \\ Correspondência \\ E. M. Souza \\ Secretaria de Estado \\ de Saúde do Distrito Federal. \\ SQN 115, Bloco "G", Apt 208, \\ Brasília, $D F$ \\ 70772-070, Brasil. \\ elzadesouza@terra.com.br
}

\section{Abstract}

The idea of health promotion predates the use of the actual term. However, the incorporation of this idea and the practice of health promotion were influenced by the Canadian health reform movement, which echoed the voices of many others who were concerned with the influence of the physical and social environment on health. This provided the basis for the World Health Organization to launch a series of conferences beginning with the Alma Ata Conference in 1977 and followed by the Ottawa Conference, from which resulted the first international document on health promotion, known as the Ottawa Charter. Although health promotion has been the subject of a wide range of studies, the concept is still not well understood and its explicit practice is limited. Health conferences have been important for keeping the notion of equity in health alive, while the gap between the rhetoric of these conferences and practice remains to be bridged. However, the rise of social epidemiology and the development of the concept of social capital for health could bring new insights into traditional epidemiology in order to narrow this gap. The purpose of this paper is to elucidate these concepts and to describe the roles they play in public health in order to stimulate further debate.

Health Promotion; Prevention \& Control; Equity
Elza Maria de Souza 1,2

Emily Grundy 2

\section{Introdução}

A idéia de promover saúde tem se tornado uma força vital no novo movimento de saúde pública, no qual a saúde é concebida também como um fenômeno social que diz respeito à qualidade de vida e capital social 1 . Elementos de capital social tais como confiança mútua, normas de reciprocidade ou solidariedade, e engajamento cívico 2,3,4 aliados ao crescimento da epidemiologia social são temas que poderão trazer novas perspectivas ao campo da saúde pública e da promoção de saúde.

\section{Conceito de promoção de saúde e o modelo biomédico}

A promoção de saúde tem sido definida como o processo que capacita a população a exercer e aumentar o controle sobre a sua saúde, sendo dessa forma relativa ao bem estar individual e coletivo (Organização Mundial da Saúde, Carta de Ottawa; 1986). A distinção entre prevenção, educação para a saúde e promoção da saúde ainda não é muito clara, mesmo para profissionais da área. Embora os limites para tais definições não sejam rígidos, a compreensão desses conceitos facilita a elaboração de estratégias para efetivação da prática de promoção de saúde. A prevenção baseia-se, geralmente, na concepção de risco ou da probabilidade de 
se tornar doente 5 . A educação para a saúde envolve a transmissão de informações relativas à saúde, visando à mudança de comportamento e à adoção de estilo de vida saudável, em que o indivíduo passa ser o principal responsável pelo seu estado de saúde. Ainda que desejáveis e necessárias, as abordagens e campanhas educativas, quando mal conduzidas, podem ter efeito deletério, uma vez que podem despertar preocupação exagerada, tornando certos "grupos de risco" em "grupos de risco em pânico" 6 . A promoção de saúde implica um processo mais abrangente e contínuo, que envolve prevenção, educação e a participação de diferentes setores da sociedade na elaboração de estratégias que permitam a efetividade da educação para a saúde. Dessa forma, a promoção transcende as atividades e as decisões individuais para tornar-se uma atividade coletiva 7.

O modelo biomédico de saúde com o seu foco principal na etiologia, diagnóstico e tratamento das doenças tem dado uma importante contribuição ao desenvolvimento da assistência médica. No entanto, essa abordagem reducionista, em geral não leva em consideração outros fatores que influenciam a saúde tais como o ambiente físico e social onde os problemas de saúde ocorrem 7,8. Considerando-se que a saúde envolve inúmeras variáveis relativas ao indivíduo e ao meio ambiente, sua interpretação exige um enfoque mais amplo, não sendo, portanto, apropriado o enfoque reducionista. O movimento de promoção de saúde veio, não para se opor, mas para preencher essa lacuna e propor uma abordagem mais estruturalista ao modelo biomédico, em que a prevenção e o tratamento das doenças, bem como a adoção de comportamento e estilo de vida saudável requerem medidas de foro ambiental, econômico, sócio-cultural e legislativo para sua efetivação 7 (Organização Mundial da Saúde, Carta de Ottawa; 1986, Organização Mundial da Saúde, Health Promotion: A Discussion Document on the Concepts and Principles; 1984).

\section{O movimento de promoção de saúde}

Do ponto de vista histórico, a promoção da saúde como idéia e como base para a elaboração de políticas sociais antecede o uso explícito da expressão. Por mais que a abordagem intervencionista tenha dominado a medicina, MacDonald 7 e Waldron ${ }^{9}$ relatam que a prevenção dos agravos à saúde não foi complemente negli- genciado ao longo do tempo, como mostra um livro intitulado, Regimen Sanitatis Salernitanum, escrito no século XIII, o qual já destacava os benefícios nutricionais e da higiene, tanto quanto a influência positiva do exercício físico, do lazer e do descanso no estado de saúde.

De acordo com Terris 10, a expressão "promoção de saúde" foi usada pela primeira vez em 1945 por Henry Sigerist, historiador médico para quem a prática médica deveria compreender três grandes princípios: a promoção da saúde, a prevenção dos agravos à saúde, o tratamento e reabilitação. Terris já apregoava que para se ter saúde é necessário um padrão de vida aceitável no qual estão incluídas condições apropriadas de trabalho, de educação, atividades culturais e de recreação. $\mathrm{O}$ autor postulava também a importância da interação intersetorial e as alianças interdisciplinares na promoção da saúde 5 . Quarenta anos depois esses mesmos princípios estavam recomendados na Carta de Ottawa (Organização Mundial da Saúde; 1986).

A concepção e a prática de promoção de saúde, todavia, foram impulsionadas a partir de 1974, quando Marc Lalonde 11, ministro da saúde canadense, publicou o documento Uma Nova Perspectiva Para a Saúde dos Canadenses (A New Perspective on the Health of Canadians), no qual foi introduzida a idéia de que um verdadeiro incremento ambiental (abordagem estruturalista) e no comportamento populacional (abordagem comportamental do estilo de vida) poderia resultar em significativa redução da morbi-mortalidade. O documento lançado por Lalonde deu ênfase a muitas vozes que já se preocupavam com a visão limitada do modelo biomédico tradicional, o qual, de alguma forma, separa o corpo da mente, a doença do doente e o doente da sociedade 12 .

Essa nova perspectiva de saúde levantada por Lalonde provocou enorme repercussão e desencadeou uma série de iniciativas lideradas pela Organização Mundial da Saúde (OMS), começando com a Assembléia de Alma Ata (Organização Mundial da Saúde; 1977), na então União Soviética, da qual resultou a Declaração de Alma Ata. Essa declaração que preconizava saúde para todos no ano 2000 e a importância da atenção básica como recomendação chave, ressaltava também a necessidade da participação comunitária e a interação intersetorial para a saúde. Elementos esses incorporados aos programas de promoção de saúde e enfatizados nos documentos elaborados durante as conferências internacionais em promoção da 
saúde. Usando esses elementos como base, a OMS lançou um programa formal de Promoção de Saúde em 1984 (Organização Mundial da Saúde, Health Promotion: A Discussion Document on the Concepts and Principles; 1984). Esse programa motivou a realização da Primeira Conferência Internacional em Promoção da Saúde em 1986, sediada por Ottawa no Canadá, da qual resultou a Carta de Ottawa (Organização Mundial da Saúde; 1986).

\section{A Carta de Ottawa}

De acordo com os preceitos da Carta de Ottawa, para se alcançar um estado real de saúde são necessários vários pré-requisitos, incluindo educação, renda adequada, justiça social e eqüidade. Para que essas condições sejam, de fato, alcançadas, o movimento de promoção de saúde tem que tornar a ação intersetorial uma prática mais tangível 13, o que requer também alianças interdisciplinares consistentes e o desenvolvimento de movimentos comunitários suficientemente fortes para influenciar as políticas públicas 14,15,16.

A Carta de Ottawa ressalta cinco áreas de ação que fornecem os fundamentos para a provisão de promoção de saúde, incluindo o desenvolvimento de políticas públicas saudáveis, a criação de ambiente sustentável, o fortalecimento da participação comunitária, o desenvolvimento de habilidades individuais e a reorientação dos serviços de saúde.

\section{Outras conferências de saúde}

Seguindo-se a Conferência de Ottawa, outras conferências foram conduzidas em diferentes países tais como a de Adelaide, Austrália (Organização Mundial da Saúde, Recomendações de Adelaide; 1988), seguindo-se a de Sundsvall, na Suécia (Organização Mundial da Saúde, The Sundsvall Statement; 1991), na qual ficou sedimentada a idéia da criação dos espaços saudáveis 17 , tanto em pequena escala como as escolas promotoras de saúde, hospitais saudáveis, unidades básica de saúde e presídios saudáveis, quanto projetos mais ambiciosos como as cidades saudáveis que, no Brasil, recebe a denominação de municípios saudáveis 5 . A Conferência de Jakarta, Indonésia (Organização Mundial da Saúde; 1997) foi a primeira em país em desenvolvimento e a primeira a incluir o setor privado nas discussões de promoção de saúde. Durante essa conferência, todos os princípios de promoção de saúde foram novamen- te ressaltados, acrescentando-se a importância da saúde do idoso. A quinta Conferência realizada no México (Organização Mundial da Saúde, Declaração do México; 2000), além de reforçar os elementos-chave na promoção de saúde, ressaltou a relevância dos aspectos científicos, sociais e políticos na promoção da saúde, bem como a necessidade de reduzir as desigualdades entre grupos e países e o papel da mulher no desenvolvimento de políticas de saúde.

Em razão das peculiaridades da América Latina e da necessidade de debater estratégias para enfrentar os problemas da região, o Ministério da Saúde da Colômbia juntamente com a Organização Pan-Americana da Saúde (OPAS) organizaram a Conferência de Bogotá, em 1992, (Organização Pan-Americana da Saúde, Programa de Promoção da Saúde; 1992). Após essa iniciativa, em 1993, foi elaborada a Carta do Caribe para a Promoção da Saúde, por ocasião da 13a Reunião dos Ministros Responsáveis pela Saúde do Caribe, sediada em Port of Spain em Trinidad e Tobago.

\section{Promoção de saúde no Brasil}

No Brasil, como em vários países da América Latina onde as desigualdades sócio-econômicas prevalecem, a promoção da saúde ainda tem pequena participação no desenvolvimento social e econômico do país. Fatores tais como manipulação de políticas públicas, interesses políticos e instabilidade política em muitas regiões prejudicam seu desenvolvimento 5 . No entanto, em 1998, o Ministério da Saúde, em ação conjunta com a OPAS, lançou um programa nacional de promoção de saúde que encoraja e divulga as atividades por meio de seu periódico Promoção da Saúde. Os programas desenvolvidos incluem o movimento dos municípios saudáveis 18,19, as Escolas promotoras de saúde 20, Hospitais saudáveis e os Eco Clubes 21. Esses últimos constituem uma iniciativa de atenção primária ao meio ambiente. O Programa "Agita São Paulo" criado em 1996 pelo Centro de Estudos do Laboratório de Aptidão Física de São Paulo constitui uma iniciativa de promoção de saúde de repercussão nacional e internacional, reconhecido pela OMS, o qual inspirou o tema "Agita Mundo" para a comemoração do Dia Mundial da Saúde em 2002 (http://www.who.int/archives/work-health day/package.en.shtml).

Como o Brasil tem dimensão continental, muitas dessas atividades ficam diluídas e, conseqüentemente pouco visíveis. Outro fator que dificulta a divulgação é a escassa literatura exis- 
tente em avaliação dessas atividades. A avaliação é parte vital para que práticas bem sucedidas possam ser difundidas e replicadas em outras localidades. O Distrito Federal vem desenvolvendo e avaliando algumas ações de promoção de saúde especialmente na área de saúde do idoso 20,22. Entretanto, a falta de incentivos para o desenvolvimento de metodologias avaliativas reconhecidas pelos meios acadêmicos e a demora na incorporação das práticas aos serviços, atrasa, consideravelmente, o seu desenvolvimento. Outro fator que obsta a divulgação de evidências baseadas na prática é a grande distância ainda existente entre serviço, ensino e pesquisa. As investigações científicas continuam muito restritas ao ambiente das universidades, o qual muitas vezes ignora a realidade de outros setores prestadores de serviços.

Em resumo, vinte e sete anos se passaram desde que a Declaração de Alma Ata foi lançada, trazendo os rudimentos de promoção de saúde, realçados posteriormente na Carta de Ottawa. Outras conferências de promoção de saúde se sucederam e novos documentos foram elaborados; porém, parece que essas conferências e os documentos delas resultantes têm servido quase que exclusivamente para não deixar fenecer a idéia da saúde para todos, uma vez que nas regiões mais pobres do planeta ainda existe uma grande distancia entre a retórica dessas conferências e a efetivação da prática de promoção da saúde. É provável, no entanto, que o crescente interesse na área da epidemiologia social, associado ao desenvolvimento de capital social, traga novas perspectivas ao campo da saúde pública, e possivelmente a concretização da idéia de saúde como um bem coletivo.

\section{Promoção de saúde e epidemiologia social}

Epidemiologia social é definida como o ramo da epidemiologia que estuda a distribuição e a influência dos fatores sociais na saúde 23 . As três últimas décadas têm mostrado um interesse crescente em entender como a sociedade e diferentes formas de organização social influenciam a saúde e o estado de bem estar 24,25. O campo da epidemiologia social traz o foco de atenção antes voltado principalmente para os o fatores de risco para a saúde para examinar, com mais profundidade, o contexto social em que eles ocorrem. Podendo assim identificar e descrever as várias condições sociais que parecem influenciar o estado de saúde das populações. Aspectos esses pouco abordados dentro da epidemiologia tradicional.
Revisões históricas mostram que a relação entre saúde e fatores sócio-econômicos tem sido documentada por vários séculos desde a Grécia Antiga, Egito e China e que essa relação, portanto, antecede o estabelecimento da epidemiologia social 23,26,27. Krieger 24 e Berkman \& Kawachi 23 mencionam estudiosos do séculos XVII e XIX que já se preocupavam com a influência dos determinantes sociais na saúde. John Graunt em 1662 estudava os efeitos das variações sociais na morbi-mortalidade na Inglaterra. Médicos como Vilherme em 183028 e Virchow em 184829 apontavam as diferenças entre classes sociais e as condições de trabalho como fatores cruciais na determinação do estado de saúde e de doença, enquanto Durkheim 30 relacionava a integração social a padrões de mortalidade, mormente o suicídio.

Vários estudos nas áreas de psicofisiologia e psiconeuroimunologia têm mostrado a influência de fatores externos (external stressors) nas respostas fisiológicas capazes de influenciar o prognóstico ou o desenvolvimento de doenças 31,32, mesmo aquelas determinada por fatores genéticos 33. Mais recentemente a hipótese da carga alostática, ou danos do estresse induzido, tem ajudado a traçar os caminhos biológicos que conectam os padrões sociais ao estado de saúde 31,34 .

Outros pesquisadores têm demonstrado a relação entre demanda psicológica e saúde 35,36, como também a influência da coesão social e saúde 37,38,39,40. Estudos mostram ainda que a qualidade de vida social é um dos mais poderosos determinantes de saúde das populações, a qual está também estreitamente associada ao grau de eqüidade na distribuição de renda $1,37,41$.

A observação de que a saúde sofre influência do meio social e físico chama a reflexão para o fato de que em relação às doenças existem modificações epidemiológicas consideráveis. Muitas doenças infecciosas dominaram o cenário e foram erradicadas, outras emergiram e atualmente as doenças não infecciosas não comunicáveis correspondem às principais causas de morte em vários países desenvolvidos e muitos países emergentes ou pobres. Mas as desigualdades sociais continuam crescendo. Esse é dos fatores de risco para a saúde que tem recebido pouca atenção. Esse padrão clama por uma abordagem epidemiológica mais consistente em que a promoção de saúde tem papel crucial 23.

Embora bem estabelecida a relação de fatores psicossociais e saúde, ainda existe uma lacuna no que se refere ao desenvolvimento e avaliação de intervenções de promoção de saúde que incluam mecanismos que promovam a coesão social. Todavia, já é reconhecido que a área de 
promoção de saúde requer grande conhecimento proveniente da evidência baseada na prática, o que implica projetos bem delineados e avaliações criteriosas que permitam a disseminação dos resultados 42 . É provável que o conceito de capital social concebido nas últimas décadas possa dar uma importante contribuição ao desenvolvimento da prática de promoção da saúde.

\section{Capital social e saúde}

Capital social diz respeito a um conjunto de elementos da organização social tais como confiança mútua, normas de reciprocidade e solidariedade (elementos cognitivos), como também o engajamento cívico e redes de associações (elementos estruturais) que facilitam a coordenação e cooperação de ações coletivas para o alcance de benefícios mútuos 40,43,44,45. Capital social, dessa forma, se refere à coesão social que resulta de uma comunidade participativa $1,46,47$.

O termo capital social ganhou notoriedade com os trabalhos dos cientistas sociais Pierre Bourdieu 48, Putnam 2,3 e Coleman 4 e é reconhecido como um importante elemento para o desempenho político e o funcionamento da democracia, para a prevenção do crime e da delinqüência e mais recentemente para a manutenção e aprimoramento do estado de saúde 1,38, $44,46,47,49,51$. Vários estudos têm comprovado que a coesão social, a existência de confiança mútua e respeito entre diferentes setores da sociedade determinam impacto positivo na saúde 1,39,40,52. Níveis crescentes de participação reduzem a exclusão social, aumentam e aprimoram o padrão de vida comunitária. Sendo assim, capital social promove uma reação individual e coletiva em direção a uma sociedade mais saudável 53,54.

Estudiosos do assunto 46,55 ressaltam, no entanto, que cuidado precisa ser tomado ao lembrar que uma extensa literatura relacionando saúde e fatores sociais há muito já existe. É preciso examinar quando o conceito de capital social é consoante com os trabalhos realizados até o momento, e se esse conceito verdadeiramente facilita o entendimento sobre a influência das relações comunitárias na saúde.

\section{Capital social: prós e contras}

Ainda que o entusiasmo em relação ao conceito e aos benefícios de capital social seja um dado concreto, não é consensual 43,53,55,56,57,58. Os autores chamam a atenção para os benefícios e os possíveis malefícios de capital social. Entre os benéficos, destacam-se o maior controle social, a provisão de suporte mútuo e todos as vantagens derivadas das organizações sociais que os membros podem obter. Contudo, capital social pode ser usado para restringir oportunidades para não associados, para exercer demanda excessiva aos associados, para restringir liberdades individuais ou reforçar o comportamento delinqüente onde essa característica for exigida pela associação como é caso do crime organizado.

Um dos grandes defensores de capital social no momento é o Banco Mundial, o qual criou um grupo temático de capital social (Social Capial Thematic Group) e está patrocinando projetos de saúde em países de baixa renda, por entender que o investimento em saúde é um meio de promover o crescimento econômico 49. Essa iniciativa surgiu da percepção de que em muitos países, as crises econômicas estavam sendo fomentadas, em parte, pelas austeras políticas adotadas pelo Banco Mundial e pelo Fundo Monetário Internacional. Dessa forma, houve a necessidade de se adotar uma postura mais democrática, na qual capital social desempenha um papel crucial no desenvolvimento econômico 55. Essas medidas, no entanto, podem ser vistas como um meio disfarçado de encorajar o capitalismo e a concentração de poder econômico sob a aparência de investimento em capital social 49 .

A utilização do conceito de capital social exige cautela, a fim de que o seu uso não seja um pretexto para esconder ideologias indesejadas ou perpetuar o "status quo" de indivíduos ou grupos com interesses políticos ou econômicos específicos.

Cuidado também deve ser tomado para evitar que o Estado, à guisa de investir em capital social deixe sob a responsabilidade dos cidadãos, ações a ele concernentes, ou, ainda, culpar comunidades de não serem saudáveis por não serem coesas. Porém, o investimento em capital social pode trazer significativas contribuições ao aumentar o poder comunitário e, dessa maneira, facilitar ações coordenadas que irão influenciar a saúde da população. É possível que o investimento em capital social seja uma alternativa de preencher a lacuna existente entre a retórica das conferências de saúde e a implementação de ações de promoção de saúde, que exigem a ação do sistema de saúde e a participação de sistemas afins.

\section{Conclusão}

A idéia de promover saúde traz novas perspectivas ao campo da epidemiologia ao propor uma abordagem mais estruturalista ao modelo biomédico. Sob o ponto de vista cientifico, no en- 
tanto, essa proposta representa um enorme desafio, particularmente em países como o Brasil, onde práticas que requerem participação e conscientização social para a redução das desigualdades sócio-econômicas e, conseqüentemente desigualdades na saúde, põem em risco interesses políticos individuais que se sobrepõem ao desenvolvimento coletivo. Mas o cres-

\section{Resumo}

A idéia de promover saúde antecede o uso explícito do termo. No entanto, a incorporação dessa idéia e da prática no campo da saúde pública foi influenciado especialmente pela reforma de saúde iniciada no Canadá, em 1974, a qual refletiu os anseios de muitos outros preocupados com influência do meio físico e social na saúde. Essa reforma serviu de base para o lançamento de várias conferências desencadeados pela Organização Mundial da Saúde, começando pela de Alma Ata em 1977, e seguida pela Conferência de Ottawa, da qual resultou o primeiro documento internacional em promoção de saúde: a Carta de Ottawa. Embora promoção da saúde esteja presente em uma grande e variada literatura, seu conceito ainda não é bem entendido e sua prática dificultada. Parece que a importância dessas conferências tem servido para não deixar fenecer a idéia de eqüidade em saúde, uma vez que os esforços para reduzir a distância entre retórica e prática continuam longe de ser alcançados. Entretanto, o crescimento da epidemiologia social associado ao conceito de capital social para saúde podem complementar os preceitos da epidemiologia tradicional e, conseqüentemente, contribuir para a efetivação do modelo estruturalista para a promoção da saúde. O propósito desse artigo é elucidar esses conceitos e descrever o papel que eles desempenham no campo da saúde pública a fim de estimular debates futuros.

Promoção da Saúde; Prevenção \& Controle; Eqüidade

\section{Colaboradores}

Os autores trabalharam juntos na seleção da revisão da literatura e na seleção das referências. E. M. Souza elaborou o primeiro rascunho, ficando E. Grundy com a responsabilidade da revisão crítica e da correção do resumo em inglês. Ambos discutiram a versão final enviada para Cadernos de Saúde Pública.

\section{Agradecimentos}

Esse artigo é parte da revisão bibliográfica do programa de doutorado em promoção da saúde que está sendo desenvolvido na London School of Hygiene and Tropical Medicine/Departament of Epidemiology and Population Health/Centre for Population Studies, Londres, Inglaterra. Programa financiado pela CAPES, Secretaria de Estado de Saúde do Distrito Federal e Centre for Populations Studies/EPH/LSHTM. cimento da epidemiologia social, possibilitando o entendimento da influência do meio físico e social no estado de saúde e a construção de capital social, facilitando a coordenação de ações para objetivos comuns, parecem abrir novos caminhos no campo da saúde pública em direção a uma população mais saudável.

\section{Referências}

1. Wilkinson RG. Unhealthy societies: the afflictions of inequality. London: Routledge; 1996.

2. Putnam RD. Boiling alone: American's decline social capital. Journal of Democracy 1995; 6: 65-78.

3. Putman RD. Making democracy work. Princeton: Princeton University Press; 1993.

4. Coleman JS. Foundations of social theory. Cambridge: Harvard University Press; 1990.

5. Ferraz ST. Cidades saudáveis: uma urbanidade para 2000. Brasília: Paralelo15; 1999.

6. Becker MH. A medical sociologist looks at health promotion. J Health Soc Behav 1993; 34:1-6.

7. MacDonald TH. Rethinking health promotion: a global approach. London: Routledge; 1998.

8. Downie RS, Tannahil C, Tannahill A. Health promotion models and values. Oxford: Oxford University Press; 1996.

9. Waldron HA. The medical role in environmental health. Oxford: Oxford University Press; 1978.

10. Terris M. Concepts of health promotion: dualities in public health theory. J Public Health Policy 1992; 13:267-76.

11. Lalonde M. A new perspective on health of Canadians. Ottawa information. Ottawa: Canadian Department of National Health and Welfare; 1974.

12. Basaglia FO. The changing culture of health and difficulties of public health to cope with it. Vienna dialogue on health policy and heath promotion - Towards a new conception of public health. Brussels: EEC Publications; 1986.

13. Kickbush I. Health promotion: a way forward. Health Promot Int 1995; 10:1-3.

14. Berckman LF, Glass T, Brissette I, Seeman TE. From social integration to health: Durkheim in the new millennium. Soc Sci Med 2000; 51:843-57.

15. Kalnins I, Hart C, Ballantyne P, Quartaro G, Love $\mathrm{R}$, Sturis G, et al. School-based community development as a health promotion strategy for children. Health Promot Int 1994; 9:269-79.

16. Chavis MD, Wandersman A. Sense of community in the urban environment: a catalyst for participation and community development. Am J Community Psychol 1990; 18:55-81.

17. Kelleher C. Evaluating health promotion in four key settings. In: Davies JK, MacDonald G, editors. Quality, evidence and effectiveness in health promotion: striving for certainties. London: Routledge; 1998. p. 47-67.

18. Gentile M. Os desafios dos municípios saudáveis. Promoção da Saúde 1999; 1:12-6.

19. Braga JM. Fazendo muito com pouco por todo o 
Brasil. Promoção da Saúde 1999; 2:19-23.

20. Souza EM. Reminiscências na promoção da saúde: uma experiência bem sucedida no Distrito Federal, Brasil. Revista de Saúde do Distrito Federal 2001; 12:35-7.

21. Terra O. Programa comunidade ativa. Promoção da Saúde 2000; 3:35-41.

22. Souza EM. Intergenerational interaction in health promotion: a qualitative study in Brazil. Rev Saúde Pública 2003; 37:463-9.

23. Berckman LF, Kawachi I. A historic framework for social epidemiology. In: Berkman L, Kawachi I, editors. Social epidemiology. Oxford: Oxford University Press; 2000. p. 3-12.

24. Krieger N. A glossary of social epidemiology. J Epidemiol Community Health 2001; 55:693-700.

25. Stansfeld SA, Fuhrer R, Shipley MJ. Types of social support as predictors of psychiatric morbidity in a cohort of British Civil Servants (Whitehall II Study). Psychol Med 1998; 28:881-92.

26. Oakes JM, Rossi PH. The measurement of SES in health research: current practice and steps toward a new approach. Soc Sci Med 2003; 56:769-84.

27. Ackerknecht EH. A short history of medicine. Baltimore: The John Hopkins University Press; 1982.

28. Villerme LR. De la mortalite dans divers quarters de la ville de Paris. Annales d'Hygiene Publique 1830; 3:294-341.

29. Virchow R. Collected essays on public health and epidemiology. Science History 1848; 1:205-20.

30. Durkheinm E. Suicide: a study in sociology. New York: Free Press; 1897.

31. Brunner E, Marmot M. Social organization, stress, and health. In: Marmot M, Wilkinsons R, editors. Social determinants of health. Oxford: Oxford University Press; 1999. p. 17-43.

32. Cannon WB. Stress and strains of homeostasis. American Journal of Medical Science 1935; 189:114.

33. Canatan D, Ratep S, Kaptan S, Cosan R. Psychosocial burden of ?-thalassaemia major in Antalya, south Turkey. Soc Sci Med 2003; 56:815-9.

34. Wadsworth M. Early life. In: Marmot M, Wilkinson RG, editors. Social determinants of health. Oxford: Oxford University Press; 1999. p. 44-63.

35. Siegrist J. Adverse health effects on high-effort/ low-reward conditions. J Occup Health Psychol 1996; 1:27-41.

36. Bosman H, Marmot MG, Hemengway H, Nicholson AC, Brunner E, Stansfeld AS. Low job control and risk of coronary heart decease in the Whitehall II (prospective cohort) study. BMJ 1997; 314: 558-65.

37. Wilkinson RG. Class mortality differentials. Income distribution and trends in poverty 1921 1981. J Soc Policy 1989; 18:307-35.

38. Marmot M, Wilkinson RG. Introduction. In: Marmot M, Wilkinson RG, editors. Social determinants of health. Oxford: University Press; 1999. p. 1-16.

39. Stansfeld SA. Social support and social cohesion. In: Marmot M, Wilkinson RG, editors. Social determinants of health. Oxford: University Press; 1999. p. 155-74.

40. Kawachi I. Social capital and community effects on population and individual health. Ann N Y Acad Sci 1999; 896:120-30.
41. Lynch JW, Smith GD, Kaplan GA, House JS. Income inequality and mortality: importance to health of individual income, psychosocial environment, or material conditions. BMJ 2000; 320: 1200-4.

42. Glass AT. Psychosocial Intervention. In: Berkman LF, Kawachi I, editors. Social epidemiology. Oxford: Oxford University Press; 2000. p. 267-305.

43. Harphan T. Measuring the social of Children. London: London South Bank University; 2003. (Working Paper 4).

44. Kawachi I, Kennedy BP, Glass R. Social capital, and self-rated health: a contextual analysis. Am J Epidemiol 1999; 89:1187-93.

45. Grootaert C. Social capital: the missing link? Social Capital Initiative. Washington DC: The World Bank; 1998.

46. Campbell C, Wood R, Moira K. Social capital and health. London: Health Education Authority; 1999.

47. Kawachi I, Berkman L. Social cohesion, social capital, and health. In: Berkman L, Kawachi I, editors. I. Social epidemiology. Oxford: Oxford University Press; 2000. p. 174-90.

48. Bordieu P. The forms of social capital. In: Richardson JG, editor. Handbook of theory and research for sociology of education. New York: Greenwood Press; 1986. p. 241-8.

49. Hawe P, Shiell A. Social capital and health promotion: a review. Soc Sci Med 2000; 51:817-55.

50. Kawachi I, Kennedy BP, Lochner K, ProthrowStith D. Social capital, income inequality, and mortality. Am J Epidemiol 1997; 87:1491-8.

51. Brehen J, Rahn W. Individual level for causes and consequences of social capital. Am J Pol Sci 1997; 41:999-1023.

52. Skrabski A. Social capital in a changing society: cross sectional associations with middle aged female and male mortality rates. J Community Health 2003; 57:114-25.

53. Baum FE, Ziersch AM. Glossary social capital. J Epidemiol Community Health 2003; 57:320-3.

54. Baum FE, Bush RA, Modra CC, Murray CJ, Cox EM, Alexander KM, et al. Epidemiology of participation: an Australian community study. J Epidemiol Community Health 2000; 54:414-23.

55. Muntaner C, Lynch J, Smith GD. Social capital and the third way in public health. Critical Public Health 2000; 10:107-24.

56. Macinko J, Starfield B. The utility of social capital in research on health determinants. Milbank Q 2001; 79:387-427.

57. Portes A. Social capital: its origins and applications in modern sociology. Annu Rev Sociol 1998; 24:1-24.

58. Putzel J. Accounting for the dark side of social capital: reading Robert Putnam on Democracy. J Int Dev 1970; 9:939-49.

Recebido em 24/Jun/2003

Versão final reapresentada em 04/Mai/2004

Aprovado em 26/Mai/2004 